\title{
Quais fatores de risco determinam a cárie dentária nos dias atuais? Uma scoping review
}

\author{
What risk factors determine tooth caries today? A scoping review
}

¿Qué factores de riesgo determinan la caries dental en la actualidad? Una scoping review

Recebido: 26/05/2021 | Revisado: 01/06/2021 | Aceito: 05/06/2021 | Publicado: 20/06/2021

\author{
Ana Beatriz Vieira da Silveira \\ ORCID: https://orcid.org/0000-0003-1162-1235 \\ Universidade de São Paulo, Brasil \\ E-mail: anab128vieira@gmail.com \\ Aluísio Eustáquio de Freitas Miranda Filho \\ ORCID: https://orcid.org/0000-0002-1348-322X \\ Universidade José do Rosário Vellano, Brasil \\ E-mail: aluisiomiranda@ hotmail.com.br \\ Nádia Carolina Teixeira Marques \\ ORCID: https://orcid.org/0000-0001-5046-2906 \\ Universidade José do Rosário Vellano, Brasil \\ E-mail: nadia.marques@unifenas.br \\ Heloisa de Sousa Gomes \\ ORCID: https://orcid.org/0000-0002-0065-9556 \\ Universidade Federal de Alfenas, Brasil \\ E-mail: hsousagomes@yahoo.com.br
}

\begin{abstract}
Resumo
Devido a sua etiologia multifatorial, a doença cárie está associada a diversos fatores, incluindo comportamentais, ambientais e socioeconômicos, hábitos alimentares, história odontológica e médica pregressa familiar, experiência prévia de cárie, bem como a carência de serviços odontológicos. Diante do grande número de fatores existentes nos dias atuais que podem estar relacionados com o surgimento da lesão e instalação da doença cárie, torna-se necessário a investigação das evidências científicas mais recentes sobre este tema. Portanto, o objetivo desta scoping review foi evidenciar a literatura científica sobre os fatores de risco relacionados atualmente com o desenvolvimento da doença cárie. Foi realizada uma pesquisa bibliográfica em Março de 2021 utilizando os descritores “Dental Caries”; "Dental Care for Children"; "Health Education, Dental" e "Epidemiology”, em bases de dados eletrônicas. Após a seleção dos artigos, os dados extraídos dos artigos selecionados foram incluídos em uma tabela padronizada, e as informações obtidas, sintetizadas em quadro para melhor interpretação dos resultados disponíveis. Apesar de existirem variados fatores predisponentes, a maioria indica que a situação socioeconômica, falta de acesso odontológico e hábitos alimentares são as condições mais predominantes ao surgimento da doença. Devido à diversidade dos artigos e conclusões encontradas, comparações são difíceis de serem bem definidas. Porém, é notório o surgimento de novos fatores de risco associados a cárie dentária, por ser uma doença complexa, de etiologia multifatorial. Apesar da variedade de fatores associados, conclui-se que os principais fatores predisponentes associados à cárie dentária são os sociodemográficos e comportamentais.
\end{abstract}

Palavras-chave: Cárie dentária; Assistência odontológica para crianças; Saúde bucal; Epidemiologia.

\begin{abstract}
Due to its multifactorial etiology, caries disease is associated with several factors, including behavioral, environmental and socioeconomic factors, eating habits, family history of dental and past medical, previous experience of caries, as well as lack of dental services. Against the large number of factors that exist today that may be related to the onset of injury and the onset of caries disease, it is necessary to investigate the most recent scientific evidence on this subject. Therefore, the aim of this scoping review was to evidence the scientific literature on the risk factors currently related to the development of caries disease. A bibliographic research was carried out in March 2021 using the descriptors "Dental Caries"" Dental Care for Children"; "Health Education, Dental" and "Epidemiology", in electronic databases. After the selection of the articles, the data extracted from the selected articles were included in a standardized table, and the information obtained, synthesized in a table for better interpretation of the available results. Although there are several predisposing factors, most indicate that socioeconomic status, lack of dental access and eating habits are the most prevalent conditions for the onset of the disease. Due to the diversity of articles and conclusions found, comparations are difficult to be well defined. However, it is notorious the emergence of new risk factors associated with dental caries, because it is a complex disease of multifactorial etiology. Despite the variety of associated factors, it is concluded that the main predisposing factors associated with dental caries are sociodemographic and behavioral factors.
\end{abstract}


Keywords: Dental caries; Dental assistance for children; Oral health; Epidemiology.

\section{Resumen}

Debido a su etiología multifactorial, la enfermedad de caries se asocia con varios factores, incluidos factores conductuales, ambientales y socioeconómicos, hábitos alimenticios, antecedentes familiares dentales y médicos, experiencia previa de caries, así como la falta de servicios dentales. Ante la gran cantidad de factores existentes en la actualidad que pueden estar relacionados con el inicio de la lesión y el inicio de la enfermedad de caries, es necesario investigar las evidencias científicas más recientes sobre este tema. Por lo tanto, el propósito de esta revisión de alcance fue resaltar la literatura científica sobre los factores de riesgo actualmente relacionados con el desarrollo de la enfermedad de caries. En marzo de 2021 se realizó una búsqueda bibliográfica utilizando los descriptores "Caries dental" "Atención dental para niños"; "Educación en Salud, Odontología" y "Epidemiología", en bases de datos electrónicas. Una vez seleccionados los artículos, los datos extraídos de los artículos seleccionados se incluyeron en una tabla estandarizada, y la información obtenida, sintetizada en una tabla para una mejor interpretación de los resultados disponibles. Aunque existen varios factores predisponentes, la mayoría indica que la situación socioeconómica, la falta de acceso odontológico y los hábitos alimentarios son las condiciones más prevalentes para el inicio de la enfermedad. Debido a la diversidad de artículos y conclusiones encontradas, las comparaciones son difíciles de definir bien. Sin embargo, es notoria la aparición de nuevos factores de riesgo asociados a la caries dental, por tratarse de una enfermedad compleja y de etiología multifactorial. A pesar de la variedad de factores asociados, se concluye que los principales factores predisponentes asociados a la caries dental son factores sociodemográficos y conductuales.

Palabras clave: Caries dental; Asistencia dental para niños; Salud bucal; Epidemiología.

\section{Introdução}

A cárie dentária é uma doença biopsicossocial complexa, decorrente de um desequilíbrio na microbiota bucal nativa (Associação Brasileira de Odontopediatria, 2020) . O primeiro relato caracterizando esta doença como multifatorial é de 1962, no qual Keys definiu os seguintes fatores relacionados ao desenvolvimento das lesões: o hospedeiro (dentes), os microrganismos e a dieta. Posteriormente, com novos estudos Newbrun adicionou um novo fator determinante: o tempo (Newbrun, 1983). Porém, observa-se que a doença cárie é ainda mais complexa do que isto, sendo considerada um problema de saúde pública até os dias atuais (Magalhães, et al. 2021) (Foulds, 2018).

Desta maneira, a avaliação do risco de cárie pode ser definida como a probabilidade de um indivíduo desenvolver a doença, de acordo com a análise da presença de fatores de risco. A partir do entendimento desse conceito, o profissional consegue atuar de maneira individualizada com abordagens educativas, preventivas e até mesmo terapêuticas. Diante disso, é imprescindível que o cirurgião-dentista conheça os fatores relacionados atualmente ao desenvolvimento da cárie dentária (Magalhães, et al. 2021).

Diversos fatores tem sido investigados a fim de se identificar uma possível relação com o aparecimento de novas lesões cariosas, como por exemplo: fatores comportamentais, ambientais (Rapôso, 2018), socioeconômicos (Ferreira, et al. 2007) (Kramer, et al. 2018) (Corrêa-Faria, et al. 2016) (Engelmann, et al. 2016), hábitos alimentares, história odontológica e médica pregressa familiar, experiência prévia de cárie (Corrêia-Faria, et al. 2016), carência de serviços odontológicos (Silveira, et al. 2015), nascimento prematuro (Diniz, et al. 2011), alta prevalência de defeitos de esmalte (Martello, et al. 2012) (Cortines, et al. 2019), deficiência proteica (Dias, Viveiros, 2014), bem como, a hipofunção das glândulas salivares (Costa, et al. 2010).

Numerosos estudos recentes relatam que a prevalência de cárie dentária e seu nível de gravidade está associado a pior qualidade de vida relacionada à saúde bucal em pré-escolares e famílias (Rajab, Abdullah, 2020), (Pesaressi, Villena, Frencken, 2020). No entanto, o impacto que esses fatores podem causar na qualidade de vida dessas crianças, e respectivas famílias, pode ser diferente em cada população (Chen, 2017). Desta forma, é de suma importância avaliar os principais fatores de risco associados a cárie, para que, a partir disso, se defina o melhor tratamento.

Sendo assim, diante do grande número de fatores existentes nos dias atuais, relacionados com o desenvolvimento da lesão e da instalação da doença cárie, justifica-se a investigação de evidências científicas recente sobre este tema. Portanto, o 
objetivo desta scoping review foi fazer um levantamento da literatura científica sobre os diversos fatores de risco existentes atualmente, relacionados com o desenvolvimento da doença cárie.

\section{Metodologia}

Trata-se de uma scoping review, que é uma revisão de literatura realizada para reconhecer um tema heterogêneo, que dificulta uma pesquisa mais precisa (Peters, et al. 2015). A pesquisa bibliográfica deste trabalho foi realizada em março de 2021, nas bases de dados eletrônicas PubMed (Public Medline ou Publisher Medline), Scielo (Scientific Eletronic Library Online) e Lilacs (Literatura Latino-Americana e do Caribe em Ciências da Saúde).

Foram usados os descritores "Cárie dentária" / "Dental caries", "Assistência odontológica para crianças" / "Dental assistance for children", "Saúde bucal" / "Oral health" e "Epidemiologia" / "Epidemiology". Na realização da busca, foram consideradas publicações atuais dos últimos 7 anos, publicadas em português, inglês e espanhol, devido à grande variedade de fatores nos estudos publicados. A busca nas bases de dados, bem como, a seleção dos artigos foi realizada por dois pesquisadores sob supervisão da pesquisadora responsável.

A seleção inicial dos artigos foi realizada através da leitura dos títulos e resumos. Os tipos de estudo como, relatos de casos, revisões, editoriais, estudos laboratoriais, e assuntos não relacionados aos fatores de risco foram excluídos. Após a seleção final dos artigos, a extração dos dados foi realizada pela leitura completa dos trabalhos, que foram incluídos em uma tabela padronizada, e as informações obtidas, sintetizadas em quadro para melhor interpretação dos resultados disponíveis.

\section{Resultados}

O fluxograma desta revisão está apresentado na Figura 1, detalhando-se o número de estudos identificados em cada etapa. De acordo com os termos utilizados durante a busca nas bases de dados eletrônicas, identificou-se inicialmente 50 referências, logo após, as duplicatas foram removidas, ficando assim 42 artigos, dos quais apenas 22 atenderam aos critérios de elegibilidade (Tabela 1).

Quinze dos 22 estudos incluídos apontam para tais fatores primordiais para o aparecimento da doença cárie, como, socioeconômicos, desigualdades, desnutrição e qualidade de vida ruim, (Restrepo-Perez, et al. 2018) (Jain et al. 2015) (Bashirian, et al. 2018) (Cianetti, et al. 2017) (Wulaerhan. et al. 2014) (Joury, et al. 2016) (Julihn et al. 2018) (Martignon et al. 2018) e (Mathur et al. 2014) (Peltzer et al. 2015) (Paula; Ambrosano; Mialhe, 2015) (Paula, et al. 2016) (Yang et al. 2016) (Bhayade, et al. 2016) (Lam et al. 2017). Além disso, é visto que o baixo nível de escolaridade dos pais interfere na condição bucal dos filhos, e o baixo desenvolvimento escolar destes é uma consequência da saúde bucal precária (Yang et al. 2016). Por outro lado, um estudo argumenta que este risco está associado à falta de fluoretação das águas (Do, LG; Ha, DH; Spencer, 2015).

Três artigos indicaram que o comportamento, associado à nutrição, como uso de carboidratos e refrigerantes na dieta, e à higiene oral precária, geram maior predisposição a doença cárie (Paisi, et al. 2018) (Biscaglia, et al. 2019) (Do, LG; Ha, DH; Spencer, 2015). Outro estudo apontou que crianças imigrantes e refugiadas também tem um maior risco de desenvolver a doença (Hoover; Vatanparast; Uswak, 2017), enquanto o estudo de Baker, et al. 2018 relatou que tipo de regime, IDH (Índice de Desenvolvimento Humano), PIB (Produto Interno Bruto) per capta também tem relação com o surgimento de cárie em crianças. 
Figura 1: Etapas de busca e seleção dos artigos da scoping review.

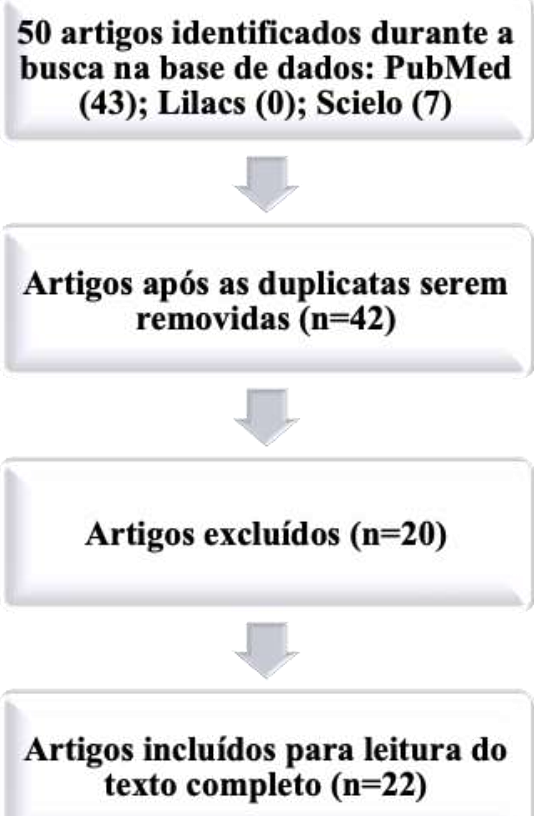

Fonte: Autores. 
Research, Society and Development, v. 10, n. 7, e24810716548, 2021

(CC BY 4.0) | ISSN 2525-3409 | DOI: http://dx.doi.org/10.33448/rsd-v10i7.16548

Tabela 1: Tabela das referências incluídas de acordo com os critérios de elegibilidade.

\begin{tabular}{|c|c|c|c|c|c|}
\hline Ano & Autor(es) & Tipo de Estudo & População de estudo & Fatores de risco estudados & Fatores de risco que foram associados à cárie \\
\hline \multirow[t]{2}{*}{2014} & Mathur et al. & Estudo transversal & Adolescentes de 12 a 15 anos. & Fatores socioeconômicos & $\begin{array}{l}\text { A área de residência é determinante e significativa para um } \\
\text { adolescente com cárie. }\end{array}$ \\
\hline & Wulaerhan et al. & Estudo transversal & 670 crianças de 3 a 5 anos de idade. & $\begin{array}{l}\text { Histórico sociodemográfico; hábitos de } \\
\text { alimentação; comportamentos relacionados à } \\
\text { higiene dental; o conhecimento geral de saúde } \\
\text { bucal dos cuidadores }\end{array}$ & $\begin{array}{l}\text { A prevalência de cárie entre crianças está relacionada com o nível } \\
\text { socioeconômico mais baixo; os comportamentos de higiene bucal } \\
\text { das crianças; o conhecimento geral de saúde bucal dos cuidadores } \\
\text { também apresentou associação significativa com a cárie dentaria. }\end{array}$ \\
\hline \multirow[t]{4}{*}{2015} & Do LG et al. & Estudo transversal & $\begin{array}{l}\text { Crianças que foram selecionadas por } \\
\text { meio de estratificação, seleção } \\
\text { amostral aleatória em 2010-2011. }\end{array}$ & $\begin{array}{l}\text { Status socioeconômico; comportamentos } \mathrm{e} \\
\text { práticas de saúde bucal; padrões alimentares; e } \\
\text { status de fluoretação. }\end{array}$ & $\begin{array}{l}\text { Crianças nas áreas não fluoretadas apresentaram prevalência } \\
\text { significativamente maior de cárie dentária. }\end{array}$ \\
\hline & Jain et al. & Estudo transversal & $\begin{array}{l}1400 \text { crianças de } 0 \text { a } 71 \text { meses, } \\
\text { registrando cárie. }\end{array}$ & $\begin{array}{l}\text { Fatores sociodemográficos; hábitos alimentares; } \\
\text { higiene } \\
\text { hábitos e características parentais. }\end{array}$ & $\begin{array}{l}\text { As variáveis significativamente associadas à cárie foram: idade, } \\
\text { localização geográfica, duração do aleitamento materno } \\
\text { mamadeira, uso de chupeta adoçada, frequência de lanches, } \\
\text { frequência de escovação, a pessoa responsável pelos cuidados de } \\
\text { saúde bucal da criança e o nível de escolaridade dos pais. }\end{array}$ \\
\hline & Paula et al. & Estudo transversal & Escolares de 12 anos de idade. & $\begin{array}{l}\text { Fatores socioeconômicos, ambiental, doméstico e } \\
\text { autopercepção } \\
\text { condições de saúde na experiência de cárie } \\
\text { dentária em crianças em idade escolar. }\end{array}$ & $\begin{array}{l}\text { Tipo de escola, renda familiar mensal, educação dos pais, } \\
\text { propriedade de casa, número de pessoas que vivem na casa, a } \\
\text { percepção dos pais sobre a saúde bucal de seus filhos e } \\
\text { autopercepção de saúde bucal dos escolares (p <0,05), foram } \\
\text { significativamente associados às piores condições de cárie } \\
\text { dentária das crianças. }\end{array}$ \\
\hline & $\begin{array}{l}\text { Peltzer; } \\
\text { Mongkolchati }\end{array}$ & $\begin{array}{l}\text { Estudo de coorte } \\
\text { prospectivo }\end{array}$ & Crianças prematuras graves. & $\begin{array}{l}\text { Fatores ambientais, comportamento de risco e } \\
\text { fatores psicológicos. Além disso, amamentar ao ir } \\
\text { para a cama, introdução de refrigerantes aos } 12 \\
\text { meses, tendo consumido com mais frequência } \\
\text { alimentos doces e menos que uma escovação } \\
\text { diária antes dos } 30 \text { meses. }\end{array}$ & $\begin{array}{l}\text { Observou-se uma taxa muito alta de cárie, e a saúde bucal pode } \\
\text { ser influenciada por fatores sociais. }\end{array}$ \\
\hline \multirow[t]{2}{*}{2016} & Paula et al. & Estudo de caso-controle & $\begin{array}{l}\text { Escolares de } 8 \text { a } 10 \text { anos da cidade de } \\
\text { Piracicaba. }\end{array}$ & $\begin{array}{l}\text { Avaliou } \quad \text { a } \text { subjetividade } \text { de } \\
\text { percepções dos pais, fatores socioeconômicos, e } \\
\text { condições clínicas orais de crianças com seus } \\
\text { rendimento escolar. }\end{array}$ & $\begin{array}{l}\text { Fatores socioeconômicos e presença das lesões de cárie, mesmo } \\
\text { tratadas, eram indicadores de risco para um mau desempenho } \\
\text { escolar. }\end{array}$ \\
\hline & Bhayade et al. & Estudo transversal & 324 crianças Anganwadis. & $\begin{array}{l}\text { Desnutrição, baixa escolaridade dos pais, práticas } \\
\text { de higiene oral e número de irmãos. }\end{array}$ & $\begin{array}{l}\text { Os estudos apontaram associações significativas entre idade, } \\
\text { desnutrição, status educacional dos pais, práticas de higiene }\end{array}$ \\
\hline
\end{tabular}


Research, Society and Development, v. 10, n. 7, e24810716548, 2021

(CC BY 4.0) | ISSN 2525-3409 | DOI: http://dx.doi.org/10.33448/rsd-v10i7.16548

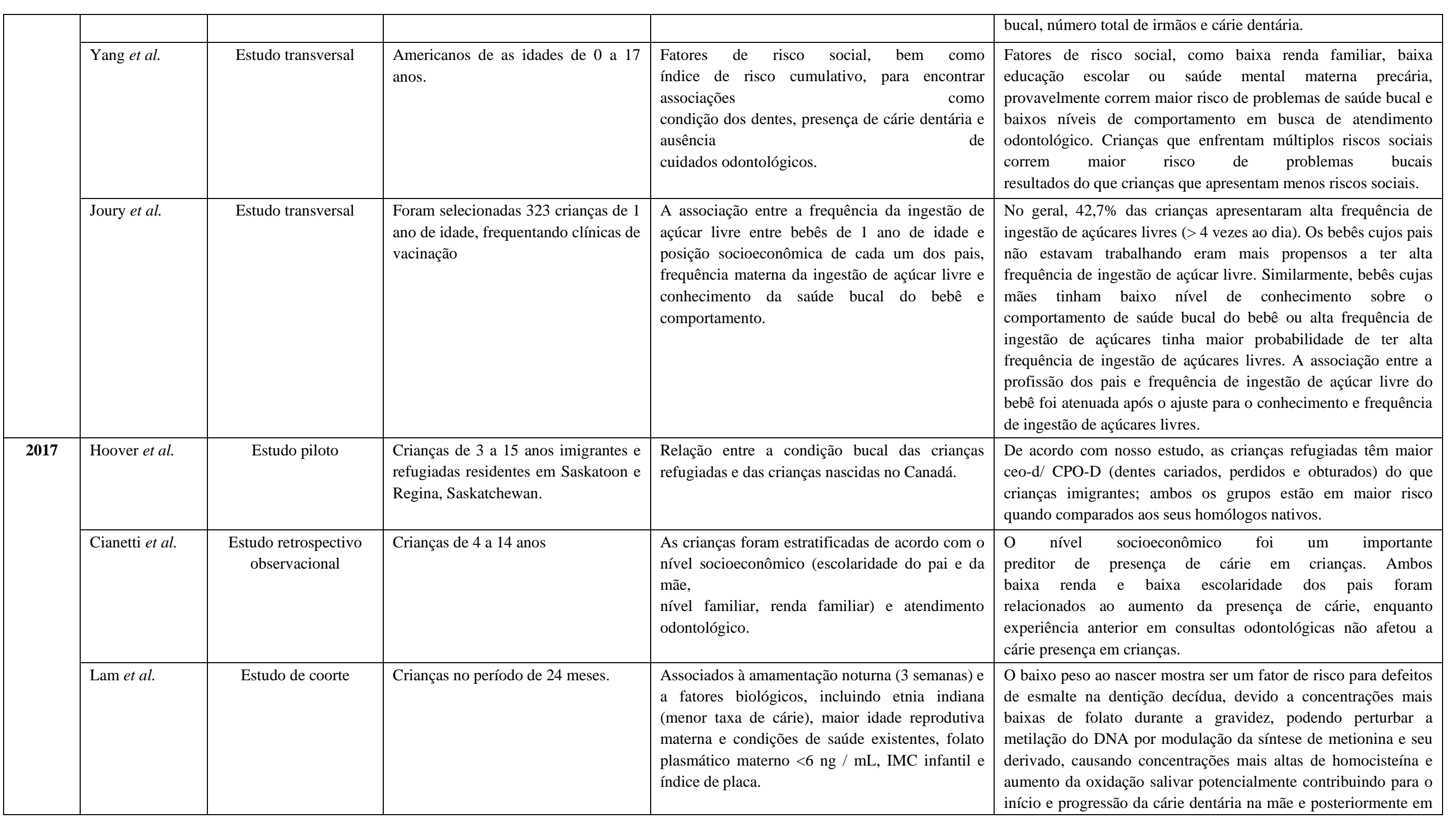


Research, Society and Development, v. 10, n. 7, e24810716548, 2021

(CC BY 4.0) | ISSN 2525-3409 | DOI: http://dx.doi.org/10.33448/rsd-v10i7.16548

\begin{tabular}{|c|c|c|c|c|c|}
\hline & & & & & bebês. \\
\hline \multirow[t]{4}{*}{2018} & Baker et al. & $\begin{array}{l}\text { Estudo longitudinal } \\
\text { cruzado }\end{array}$ & Crianças de 8 a 15 anos em 11 países. & $\begin{array}{l}\text { Qualidade de vida relacionada à saúde bucal e } \\
\text { quatro } \\
\text { determinantes } \quad \text { (governança, } \\
\text { macroeconômica, política pública e política } \\
\text { social). }\end{array}$ & $\begin{array}{l}\text { O tipo de regime político, a quantidade de governança (por } \\
\text { exemplo, direito, responsabilidade), PIB per capita, taxa de } \\
\text { emprego, desigualdade de renda, tipo de regime de bem-estar, } \\
\text { índice de desenvolvimento humano, gastos governamentais em } \\
\text { saúde, bem como gastos com saúde (privados) os gastos dos } \\
\text { cidadãos estavam todos associados à saúde bucal das crianças. }\end{array}$ \\
\hline & Bashirian et al. & Estudo transversal & Crianças iranianas de 6 a 12 anos & $\begin{array}{l}\text { Dados sobre educação e ocupação dos pais, } \\
\text { bairro, dor dentária no último ano e escovação de } \\
\text { dentes } \\
\text { os hábitos sob supervisão dos pais. }\end{array}$ & $\begin{array}{l}\text { O maior ceo-d foi observado em crianças do ensino fundamental } \\
\text { de } 7 \text { a } 8 \text { anos de idade } 6,53(4,37) \text { e o maior CPOD (dentes } \\
\text { cariados, perdidos e obturados) e placa dentária foi em crianças } \\
\text { de } 12 \text { anos registrados como } 1,17(1,77) \text { e } 51,97 \text { ( } 25,86 \text {, } \\
\text { respectivamente. A proporção de dentes cariados em } 7 \text { anos de } \\
\text { idade e a escolaridade baseada no índice ceo-d foi de } 80,36 \% \text {, } \\
\text { além disso, a proporção no ensino fundamental de } 12 \text { anos foi de } \\
40,17 \% \\
\text { com base no índice CPO-D. Idade, sexo e dor dentária no último } \\
\text { ano foram significativamente associados ao CPOD e ceo-d. A } \\
\text { chance de desenvolver cárie foi de } 1,72 \text { vezes maior nos } \\
\text { estudantes que relataram dor dentária com frequência do que } \\
\text { naqueles que não apresentaram. A idade foi significativamente } \\
\text { correlacionada com placa dentária de tal forma que o Índice de } \\
\text { Placa aumentou } 2,44 \text { vezes por aumento de um ano na idade (p } \\
<0,001 \text { ). }\end{array}$ \\
\hline & Julihn et al. & Estudo transversal & $\begin{array}{l}\text { Crianças que nascidas de } 2000 \text { a } \\
2003 \text {. }\end{array}$ & $\begin{array}{l}\text { Condições socioeconômicas, saúde materna e } \\
\text { comportamento materno-sanitário foram } \\
\text { extraídos do registro nacional sueco. }\end{array}$ & 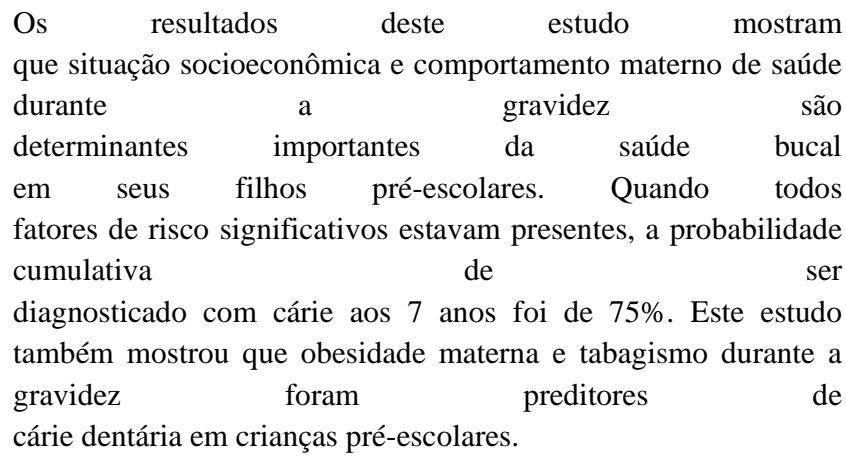 \\
\hline & Martignon et al. & Estudo transversal & $\begin{array}{l}316 \text { crianças de } 8 \text { a } 71 \text { meses de } \\
\text { idade. }\end{array}$ & $\begin{array}{l}\text { Qualidade de vida, } \begin{array}{r}\text { determinantes } \\
\text { socioeconômicos, acesso a atendimento } \\
\text { odontológico, práticas de saúde bucal, dieta e }\end{array}\end{array}$ & $\begin{array}{l}\text { Foram encontradas diferenças significantes entre as crianças com } \\
\text { e sem qualquer nível de experiencia de cárie em esmalte ou } \\
\text { dentina e as variáveis idade e nutrição. Foi encontrada também }\end{array}$ \\
\hline
\end{tabular}


Research, Society and Development, v. 10, n. 7, e24810716548, 2021

(CC BY 4.0) | ISSN 2525-3409 | DOI: http://dx.doi.org/10.33448/rsd-v10i7.16548

\begin{tabular}{|c|c|c|c|c|c|}
\hline & & & & estado nutricional. & $\begin{array}{l}\text { uma associação estatisticamente significante somente com o } \\
\text { modelo ajustado de acesso a saúde bucal para os pais com } \\
\text { escolaridade, com crianças com presença de experiencia de cárie } \\
\text { relatando } 1,9 \text { vezes mais com os pais com nível de escolaridade } \\
\text { primária X ensino médio. }\end{array}$ \\
\hline & Paisi et al. & Estudo transversal & $\begin{array}{l}\text { Crianças de quatro a seis anos de } \\
\text { idade. }\end{array}$ & $\begin{array}{l}\text { Características demográficas, renda familiar, } \\
\text { hábitos alimentares. }\end{array}$ & $\begin{array}{l}\text { Nem os escores } \mathrm{z} \text { da circunferência da cintura nem do IMC } \\
\text { mostraram ser significativamente associados à cárie dentária. } \\
\text { Entre as características examinadas do bairro, o percentual de } \\
\text { pessoas dependentes de benefícios teve um impacto significativo } \\
\text { nas taxas de cárie ( } p<0,05 \text { ). Total anual da família e a renda } \\
\text { estava inversamente relacionada ao risco de cárie e o nível de } \\
\text { escolaridade dos pais afetava a frequência de escovação dos } \\
\text { filhos. }\end{array}$ \\
\hline & Perez et al. & $\begin{array}{l}\text { Estudo descritivo } \\
\text { quantitativo }\end{array}$ & Famílias de pré-escolares. & $\begin{array}{l}\text { Foram avaliados fatores determinantes sociais, } \\
\text { aspectos econômicos, ambientais e nutricionais, } \\
\text { condições das pessoas e seu relacionamento com } \\
\text { o estado de saúde bucal. }\end{array}$ & $\begin{array}{l}\text { Havia uma relação entre a presença de cárie e o tipo de problemas } \\
\text { de saúde bucal referidos pelos pais, relação de parentesco com } \\
\text { cuidador, visitas ao serviço de saúde bucal e disponibilidade de } \\
\text { espaço para a escova de dentes }(\mathrm{p}<0,05) \text {. }\end{array}$ \\
\hline & Caruso et al. & $\begin{array}{l}\text { Estudo de caso-controle } \\
\text { observacional }\end{array}$ & $\begin{array}{l}122 \text { crianças italianas com idade entre } \\
3 \text { e } 6 \text { anos. }\end{array}$ & $\begin{array}{l}\text { Idade, níveis de cortisol salivar e escolaridade dos } \\
\text { pais. }\end{array}$ & $\begin{array}{l}\text { Os presentes achados apoiam a hipótese de que a cárie está } \\
\text { principalmente correlacionada com níveis elevados de cortisol } \\
\text { salivar. A experiência de cárie dentária em crianças também foi } \\
\text { associada positivamente com tártaro, placa bacteriana e idade. }\end{array}$ \\
\hline 2019 & Biscaglia et al. & Estudo transversal & $\begin{array}{l}\text { Estudantes de } 12 \text { anos de idade nas } \\
\text { escolas da UNRWA em cinco áreas } \\
\text { de } \\
\text { (Jordânia, Líbano, Síria, Faixão de } \\
\text { Gaza e Cisjordânia). }\end{array}$ & $\begin{array}{l}\text { Hábitos alimentares - consumo de refrigerantes, } \\
\text { frequência das refeições, gênero, hábitos de } \\
\text { higiene bucal, escolaridade da mãe. }\end{array}$ & $\begin{array}{l}\text { Ambas as pesquisas identificaram determinantes } \\
\text { comportamentais para cárie dentária, particularmente hábitos } \\
\text { alimentares, como consumo de refrigerantes. A saúde gengival } \\
\text { também mostrou diferenças estatísticas entre os campos. }\end{array}$ \\
\hline 2020 & $\begin{array}{l}\text { Gemma } \\
\text { Devenish et al. }\end{array}$ & Estudo de coortel & Crianças entre $2-3$ anos de idade & $\begin{array}{l}\text { Práticas de amamentação, fatores } \\
\text { sociodemográficos e ingestão de açúcares livres. }\end{array}$ & $\begin{array}{l}\text { Não houve associação independente entre amamentar além de } 1 \\
\text { ano de idade e CLPE, ou entre amamentar para dormir e } \\
\text { CLPE. Os únicos fatores independentemente associados à CEC } \\
\text { foram ingestão elevada de açúcares livres (PR 1,97, IC 95\%: } \\
1,13,3,44 \text { ) e maior desvantagem socioeconômica (PR 2,15, IC } \\
95 \%: 1,08,4,28 \text { ). }\end{array}$ \\
\hline
\end{tabular}

Fonte: Autores. 


\section{Discussão}

A cárie dentária ainda é uma das principais doenças bucais que acometem diferentes populações. O primeiro relato caracterizando esta doença como multifatorial é de 1962, no qual Keys definiu os seguintes fatores relacionados ao desenvolvimento das lesões: o hospedeiro (dentes), os microrganismos (biofilme), e a dieta (carboidratos fermentáveis) (Magalhães, et al. 2021). Posteriormente, Newbrun adicionou o fator tempo como um novo fator determinante para tal doença. Porém, durante esta revisão, vinte e dois artigos foram incluídos, e desta forma, foi observado que a doença cárie é muito mais abrangente e complexa do que isto, incluindo a existência de uma grande diversidade de fatores de risco relacionados a cárie dentária, ainda considerada um problema de saúde pública (Newbrun, 1983).

A condição socioeconômica foi apontada por grande parte dos autores como um fator associado à cárie dentária. Julihn et al. (2018), Martignon et al. (2018) e Mathur et al. (2014), concluíram que fatores socioeconômicos e o comportamento de saúde durante a gravidez são determinantes importantes da saúde bucal em seus filhos pré-escolares. Foi observado também que a presença de cárie foi maior em crianças onde o nível educacional dos pais era mais baixo e em crianças situadas a uma menor renda familiar (Cianetti, et al. 2017) (Wulaerhan. et al. 2014). Em uma pesquisa realizada por Yang et al. (2016), nos Estados Unidos (EUA), foi apontado que certos fatores de risco sociais, tais como baixa renda familiar e mães com saúde mental comprometida, provavelmente também aumentam o risco de problemas de saúde bucal em crianças americanas. Corroborando com estes estudos, observa-se que os fatores socioeconômicos além do tipo de escola frequentada pela criança, a renda familiar mensal, a educação dos pais, o número de pessoas que vivem na casa, e até mesmo a percepção dos pais sobre a saúde bucal de seus filhos escolares, além de estarem significativamente associadas às piores condições de cárie dentária, reduzem o rendimento escolar das crianças brasileiras (Paula; Ambrosano; Mialhe, 2015) (Paula, et al. 2016).

Crianças em idade escolar no ensino fundamental que vivem no subúrbio tiveram um maior número de dentes decíduos e permanentes cariados, em comparação com as crianças que vivem na região central da cidade. Uma possível justificativa para essa associação é o fato de que crianças que vivem no subúrbio podem apresentar dificuldades no acesso a serviços odontológicos, provavelmente devido à baixa condição socioeconômica (Bashirian, et al. 2018) (Devenish, et al. 2020). Além disso, Perez et al. (2018) salientam que, viver em áreas urbanas, ou seja, áreas povoadas da cidade, permite um melhor acesso a infraestrutura adequada para a saúde e serviços de educação. Por outro lado, Jain et al. (2015), explicam que a maior prevalência de lesões cariosas em crianças que moram em zona rural está mais relacionada com um menor uso de creme dental ou escova, visitas menos frequentes a um dentista, além da ingestão de comida açucarada mais frequente. É também importante ressaltar que essas regiões, muitas vezes, são desprovidas do suprimento de ações fluoretadas para a população, acarretando, dessa forma, a maior prevalência de cárie dentária (Do, LG; Ha, DH; Spencer, 2015).

Os hábitos alimentares e comportamentais têm sido discutidos como as principais vias de saúde bucal precária (Biscaglia, ei al. 2029). Peltzer et al. (2015) relataram que o fator nutricional e comportamental, como por exemplo, dormir com mamadeira antes dos 30 meses e introdução de refrigerantes antes dos 12 meses, aumenta significativamente a prevalência de cárie. Além disso, Paisi et al. (2018) encontraram que obesidade materna é preditora de cárie dentária em crianças préescolares, no entanto, curiosamente, o fato de a criança ser obesa não justificou o aparecimento de lesões de cárie.

Levando isso em consideração, encontra-se uma importância significativa em investigar a história médica da mãe e da criança, principalmente no momento do parto. Lam et al. (2017) mostraram que o baixo peso ao nascer pode ser um fator de risco para defeitos de esmalte na dentição decídua, uma vez que concentrações mais baixas de fosfato durante a gravidez influenciam na metilação do DNA por modulação da síntese de metionina e seu derivado, causando assim concentrações mais altas de homocisteína e aumento da oxidação salivar, o que contribui para o início e progressão da cárie dentária na mãe e posteriormente em bebês (Wulaerhan, et al. 2014). 
Outro fator evidente, foi o fato de que em residências onde responsáveis não estavam inseridos no mercado de trabalho, os bebês tendiam a apresentar maiores chances de alta frequência de ingestão de açúcar livre, além disso, as mães que tinham baixo nível de conhecimento de saúde bucal também possuíam maior probabilidade de ter uma periodicidade na ingestão de açúcares livres (Joury, et al. 2016).

No estudo recente de Baker et al. (2018), envolvendo a avaliação de 11 países, demonstrou-se que informações relevantes, no qual até mesmo o tipo de regime político, a quantidade de governança (por exemplo, direito, responsabilidade), PIB per capita, taxa de emprego, desigualdade de renda, índice de desenvolvimento humano, gastos governamentais em saúde estavam associados à saúde bucal das crianças. Grande parte deste estudo concentra-se em regimes de bem-estar social onde aqueles com políticas mais generosas, universais e redistributivas têm melhor saúde da população do que regimes menos redistributivos, evidenciando mais uma vez que o regime de bem-estar de um país deve envolver o investimento na educação e saúde, combatendo um forte fator determinante: a desigualdade social.

Recentemente, um novo fator de risco tem sido associado a experiência de cárie por Caruso et al. (2018), em que apontam uma associação de cárie dentária com o nível de cortisol, placa dentária, idade e níveis elevados de cálculo. Apoiando principalmente a hipótese de que a cárie dentária está correlacionada com estresse. Corroborando com estes resultados, uma outra pesquisa realizada no Canadá (Hoover; Vatanparast; Uswak, 2017) mostra que imigrantes e refugiados têm alta nos escores de CPOD, devido outras prioridades e preocupações, como o estresse associado à adaptação a uma nova cultura, aprender inglês como segunda língua, questões com transporte, finanças e encontrar escolas para as crianças virem antes das necessidades de saúde bucal.

É relevante ressaltar as limitações apresentadas no estudo, já que se trata de um Scoping review e não uma revisão sistemática, abordando, desta forma, um compilado de diferentes tipos de estudos. Além disso, a determinação dos fatores de riscos relacionados à carie dentária é muito extensa, assim, os fatores apresentados pelos autores podem estar inseridos em diferentes graus de evidência, tendo uns evidência mais forte e outros mais baixa, além daqueles que não foram supracitados pelos autores destacados neste estudo. Entretanto, o objetivo desta revisão foi apenas apresentar os fatores de risco e não investigar o nível de evidência que os diferentes estudos apresentam.

Em suma, é possível observar a diversidade de fatores etiológicos relacionados a cárie dentária, mostrando que a prevalência e a incidência de cárie são altamente influenciadas por uma série de fatores de risco, como idade, nível socioeconômico, desnutrição, status educacional dos pais, práticas de higiene bucal, número total de irmãos, entre outros (Bhayade, et al. 2016). Aliás, é interessante levantar a questão de que há outros fatores de risco de forte evidência que não foram citados pelos artigos selecionados para esta revisão, como por exemplo, a experiência prévia de cárie dentária, que tem um forte poder preditivo ao aparecimento de novas lesões.

\section{Conclusão}

Devido à diversidade dos artigos e conclusões encontradas, comparações são difíceis de serem bem definidas. Porém, é notório o surgimento de novos fatores de risco associados a cárie dentária por ser uma doença complexa e de etiologia multifatorial. Apesar da variedade de fatores associados, conclui-se que os principais fatores predisponentes associados à cárie dentária são os sociodemográficos e comportamentais.

Considerando isso, bem como o fato da cárie dentária ainda ser o problema de saúde bucal que mais acomete a população mundial, sugere-se o desenvolvimento de novos estudos epidemiológicos bem delineados abrangendo determinado assunto. Dessa forma, uma evidência científica mais forte poderá melhor nortear os cirurgiões-dentistas quanto a avaliação e o controle dos fatores de risco da doença cárie nos seus pacientes. 


\section{Referências}

Associação Brasileira de Odontopediatria. Diretrizes para Procedimentos Clínicos em Odontopediatria. Santos.

Baker, S. R., Foster Page, L., Thomson, W. M., Broomhead, T., Bekes, K., Benson, P. E., \& Gibson, B. J. (2018). Structural determinants and children's oral health: a cross-national study. Journal of dental research, 97(10), 1129-1136.

Bashirian, S., Shirahmadi, S., Seyedzadeh-Sabounchi, S., Soltanian, A. R., Karimi-Shahanjarini, A., \& Vahdatinia, F. (2018). Association of caries experience and dental plaque with sociodemographic characteristics in elementary school-aged children: a cross-sectional study. BMC oral health, 18(1), 1-12.

Bhayade, S. S., Mittal, R., Chandak, S., \& Bhondey, A. (2016). Assessment of social, demographic determinants and oral hygiene practices in relation to dental caries among the children attending Anganwadis of Hingna, Nagpur. Journal of Indian Society of Pedodontics and Preventive Dentistry, $34(2), 124$.

Biscaglia, L., di Caccamo, P., Terrenato, I., Arrica, M. A., Seita, A., \& Campus, G. (2019). Oral health status and caries trend among 12-year old Palestine refugee students: results from the UNRWA's oral health surveys 2011 and 2016. BMC oral health, 19(1), 1-10.

Caruso, S., Gatto, R., Cinque, B., Cifone, M. G., \& Mattei, A. (2018). Association between salivary cortisol level and caries in early childhood. European journal of paediatric dentistry, 19(1), 10-15.

Chen, K. J., Gao, S. S., Duangthip, D., Li, S. K. Y., Lo, E. C. M., \& Chu, C. H. (2017). Dental caries status and its associated factors among 5-year-old Hong Kong children: a cross-sectional study. BMC oral health, 17(1), 1-8.

Cianetti, S., Lombardo, G., Lupatelli, E., Rossi, G., Abraha, I., Pagano, S., \& Paglia, L. (2017). Dental caries, parents educational level, family income and dental service attendance among children in Italy. Eur J Paediatr Dent, 18(1), 15-8.

Correa-Faria, P., Paixao-Goncalves, S., Paiva, S. M., \& Pordeus, I. A. (2016). Incidence of dental caries in primary dentition and risk factors: a longitudinal study. Brazilian oral research, 30(1).

Cortines, A. A. D. O., Corrêa-Faria, P., Paulsson, L., Costa, P. S., \& Costa, L. R. (2019). Developmental defects of enamel in the deciduous incisors of infants born preterm: Prospective cohort. Oral diseases, 25(2), 543-549.

Costa, D. P., Mota, A. C. D. M., Bruno, G. D. B., Almeida, M. E. L. D., \& Fonteles, C. S. R. (2010). Desnutrição energético-protéica e cárie dentária na primeira infância. Revista de Nutrição, 23(1), 119-126.

Devenish, G., Mukhtar, A., Begley, A., Spencer, A. J., Thomson, W. M., Ha, D., \& Scott, J. A. (2020). Early childhood feeding practices and dental caries among Australian preschoolers. The American journal of clinical nutrition, 111(4), 821-828.

Dias, T., \& Viveiros, I. (2014). Associação entre Cárie Dentária e Obesidade numa Amostra da População Pediátrica em Vila Franca do Campo. Revista Nutrícias, (23), 14-17.

Diniz, M. B., Coldebella, C. R., Zuanon, A. C. C., \& Cordeiro, R. D. C. L. (2011). Oral abnormalities in preterm and low birth weight infants: the importance of the relationship between pediatricians and pediatric dentists. Revista Paulista de Pediatria, 29(3), 440-453.

Do, L. G., Ha, D. H., \& Spencer, A. J. (2015). Factors attributable for the prevalence of dental caries in Queensland children. Community dentistry and oral epidemiology, 43(5), 397-405.

Engelmann, J. L., Tomazoni, F., Oliveira, M. D. M., \& Ardenghi, T. M. (2016). Association between dental caries and socioeconomic factors in schoolchildren-a multilevel analysis. Brazilian dental journal, 27(1), 72-78.

Ferreira, S. H., Beria, J. U., Kramer, P. F., Feldens, E. G., \& Feldens, C. A. (2007). Dental caries in 0-to 5-year-old Brazilian children: prevalence, severity, and associated factors. International Journal of Paediatric Dentistry, 17(4), 289-296.

Foulds, H. (2017). Developmental defects of enamel and caries in primary teeth. Evidence-based dentistry, 18(3), 72-73.

Hoover, J., Vatanparast, H., \& Uswak, G. (2017). Risk determinants of dental caries and oral hygiene status in 3-15 year-old recent immigrant and refugee children in Saskatchewan, Canada: a pilot study. Journal of immigrant and minority health, 19(6), 1315-1321.

Jain, M., Namdev, R., Bodh, M., Dutta, S., Singhal, P., \& Kumar, A. (2015). Social and behavioral determinants for early childhood caries among preschool children in India. Journal of dental research, dental clinics, dental prospects, 9(2), 115.

Joury, E., Khairallah, M., Sabbah, W., Elias, K., \& Bedi, R. (2016). Inequalities in the frequency of free sugars intake among Syrian 1-year-old infants: a cross-sectional study. BMC oral health, 16(1), 1-7.

Julihn, A., Soares, F. C., Hjern, A., \& Dahllöf, G. (2018). Socioeconomic determinants, maternal health, and caries in young children. JDR Clinical \& Translational Research, 3(4), 395-404.

Kramer, A. C. A., Petzold, M., Hakeberg, M., \& Östberg, A. L. (2018). Multiple socioeconomic factors and dental caries in Swedish children and adolescents. Caries research, 52(1-2), 42-50.

Lam, C. U., Khin, L. W., Kalhan, A. C., Yee, R., Lee, Y. S., Chong, M. F., \& Hsu, C. Y. (2017). Identification of caries risk determinants in toddlers: results of the GUSTO birth cohort study. Caries research, 51(4), 271-282.

Magalhães, A.C., Rios, D., Wang, L., \& Buzalaf, M.A.R.B., Cariologia: da base à clínica/ [Alberto Carlos Botazzo Delbem] - 1. Ed - Barueri [SP]: Manole, 2021. ISBN: 9786555761078 . 
Research, Society and Development, v. 10, n. 7, e24810716548, 2021

(CC BY 4.0) | ISSN 2525-3409 | DOI: http://dx.doi.org/10.33448/rsd-v10i7.16548

Martello, R. P., Junqueira, T. P., \& Leite, I. C. G. (2012). Cárie dentária e fatores associados em crianças com três anos de idade cadastradas em Unidades de Saúde da Família do Município de Rondonópolis, Mato Grosso, Brasil. Epidemiologia e Serviços de Saúde, 21(1), 99-108.

Martignon, S., Usuga-Vacca, M., Cortés, F., Cortes, A., Gamboa, L. F., Jacome-Lievano, S., \& Ramos, N. (2018). Risk factors for early childhood caries experience expressed by ICDAS criteria in Anapoima, Colombia: a cross-sectional study. Acta odontologica latinoamericana: AOL, 31(1), 58-66.

Mathur, M. R., Tsakos, G., Millett, C., Arora, M., \& Watt, R. (2014). Socioeconomic inequalities in dental caries and their determinants in adolescents in New Delhi, India. BMJ open, 4(12).

Newbrun, E. (1983). Microflora. Cariology, 2nd ed., Baltimore: Williams and Wilkins, 50-85.

Paisi, M., Kay, E., Kaimi, I., Witton, R., Nelder, R., Potterton, R., \& Lapthorne, D. (2018). Obesity and caries in four-to-six year old English children: a crosssectional study. BMC Public Health, 18(1), 1-9.

Paula, J. S. D., Ambrosano, G. M. B., \& Mialhe, F. L. (2015). The impact of social determinants on schoolchildren's oral health in Brazil. Brazilian oral research, 29(1).

Paula, J. S., Lisboa, C. M., de Castro Meneghim, M., Pereira, A. C., Ambrosano, G. M. B., \& Mialhe, F. L. (2016). School performance and oral health conditions: analysis of the impact mediated by socio-economic factors. International journal of paediatric dentistry, 26(1), 52-59.

Peltzer, K., \& Mongkolchati, A. (2015). Severe early childhood caries and social determinants in three-year-old children from Northern Thailand: a birth cohort study. BMC Oral Health, 15(1), 1-7.

Pesaressi, E., Villena, R. S., \& Frencken, J. E. (2020). Dental caries and oral health-related quality of life of 3-year-olds living in Lima, Peru. International journal of paediatric dentistry, 30(1), 57-65.

Peters, M. D., Godfrey, C. M., Khalil, H., McInerney, P., Parker, D., \& Soares, C. B. (2015). Guidance for conducting systematic scoping reviews. JBI Evidence Implementation, 13(3), 141-146.

Pitts, N. B., Baez, R. J., Diaz-Guillory, C., Donly, K. J., Feldens, C. A., McGrath, C., \& Twetman, S. (2019). Early childhood caries: IAPD Bangkok declaration. Journal of dentistry for children (Chicago, Ill.), 86(2), 72.

Rajab, L. D., \& Abdullah, R. B. (2020). Impact of dental caries on the quality of life of preschool children and families in Amman, Jordan. Oral Health Prev. Dent, 18, 571-582.

Rapôso, N. M. L. Cárie na primeira infância e fatores associados em pré-escolares de duas creches distintas: estudo transversal. (2018) [Dissertação].

Restrepo-Perez, L. F., Usuga-Vacca, M., Cortes, A., Rodríguez-Gómez, J. C., Ortega-Mora, J. F., Roa-López, C. A., \& Martignon, S. (2018). Relationship between family-related social determinants and dental caries in preschoolers from Anapoima, Cundinamarca. Revista Facultad de Odontología Universidad de Antioquia, 30(1), 55-66.

Silveira, M. F., Freire, R. S., Nepomuceno, M. O., Martins, A. M. E. D. B. L., \& Marcopito, L. F. (2015). Cárie dentária e fatores associados entre adolescentes no norte do estado de Minas Gerais, Brasil: uma análise hierarquizada. Ciência \& Saúde Coletiva, 20, 3351-3364.

Wulaerhan, J., Abudureyimu, A., Bao, X. L., \& Zhao, J. (2014). Risk determinants associated with early childhood caries in Uygur children: a preschool-based cross-sectional study. BMC oral health, 14(1), 1-8.

Yang, A. J., Gromoske, A. N., Olson, M. A., \& Chaffin, J. G. (2016). Single and cumulative relations of social risk factors with children's dental health and care-utilization within regions of the United States. Maternal and child health journal, 20(3), 495-506. 